\title{
MRI diagnosis of carpal boss and comparison with radiography
}

M. Mespreuve ${ }^{1,2}$, L. De Smet ${ }^{3}$, K. De Cuyper ${ }^{1}$, K. Waked ${ }^{4}$, F. Vanhoenacker ${ }^{1,2,5}$

From:

Department of Radiology of St.-Maarten General Hospital, Leopoldstraat 2, 2800 Mechelen, Belgium ${ }^{1}$,

Department of Radiology of Ghent University, De Pintelaan 185, 9000 Ghent, Belgium², Orthopaedic and Hand Surgery of Leuven University, Herestraat 49, 3000 Leuven, Belgium ${ }^{3}$ Department of Surgery of Ghent University, De Pintelaan 185, 9000 Ghent, Belgium ${ }^{4}$, and

Department of Radiology of Antwerp University, Wilrijkstraat 10, 2650 Edegem - Antwerp, Belgium ${ }^{5}$.

First and corresponding author:

Prof. Dr. Marc Mespreuve, MD, PhD （ marc.mespreuve@skynet.be )

Co-authors:

Prof. Dr. Luc De Smet, MD, PhD （ luc.desmet@uzleuven.be )

Dr. Kristof De Cuper, MD （ kristof.de.cuyper@emmaus.be )

Dr. Karl Waked, MD （

Prof. Dr. Filip Vanhoenacker, MD, PhD （ filip.vanhoenacker@telenet.be ) 


\begin{abstract}
Background: A carpal boss is a potentially painful bony mass in the region of the second or third carpometacarpal joint. The combination of clinical examination and radiography is usually sufficient for the diagnosis.
\end{abstract}

Purpose: To determine whether MRI examination of the quadrangular joint can assist the diagnosis of persistent pain near a carpal boss.

Material and Methods: 57 patients with a carpal boss were retrospectively reviewed using MRI and conventional radiographs and compared to an asymptomatic control group.

Results: MRI demonstrated a variable morphology and a variety of bone and soft tissue abnormalities associated with carpal boss. Bone marrow edema around the quadrangular joint shows a significant correlation (Fischer 's exact test: $p<0.001$ ) and a positive correlation (Pearson 's test $r=0.632$, significant at the 0.01 level (two-tailed)) with a painful carpal boss. Conclusion: MRI offers detailed examination of bone and soft tissue abnormalities associated with a carpal boss. Local bone marrow edema strongly correlates with a painful carpal boss.

\title{
Keywords
}

Carpal boss, os styloideum, radiography, MRI

\section{Introduction}

A carpal boss - initially described by Fiolle (1) - is a bony prominence at the dorsal side of the second or third carpometacarpal joint. Carpal boss is often seen on imaging both in symptomatic and asymptomatic patients. Patients typically present with a local swelling and /or pain. The etiology is still unclear. 
Radiography and especially CT can analyze the bony morphology, but fail to correlate with the experienced pain. US can add information to illustrate most of the soft tissues lesions around the quadrangular joint. The presence of bony abnormalities alone usually does not allow to confirm or differentiate symptomatic findings, neither does their absence allows for exclusion of carpal boss syndrome.

Treatment is usually conservative (activity modification, anti-inflammatory analgesics, and eventually a wrist splint) and in case of persistent pain a long acting steroid can be injected. In up to $80 \%$ of the patients the pain will diminish over time. When conservative treatment fails, excision of the bony abnormality, the arthritic components, and the soft tissue swelling is considered. The exact location and morphology of the carpal boss can allow for more limited resection or shaving in order to reduce the risk of post-operative instability due to ligamentous injury.

Our primary goal was to review the MRI findings in a large series of patients and to define if there is a (potential) role of MRI in the diagnosis and selection of symptomatic cases.

\section{Material and Methods}

In this retrospective cohort study two musculoskeletal radiologists (respectively with 30 and 7 years of experience) blindly reviewed a series of 114 patients twice, with an interval of two months. Between February $11^{\text {th }} 2009$ and December $22^{\text {nd }} 2014$,they were referred to the MRI department for a wrist examination. A subgroup of 57 patients (37 males: 17 right and 20 left wrists and 20 females: 9 right and 11 left wrists) was referred with a clinical complaint of carpal boss (local swelling and /or pain at the second or third carpometacarpal joint).

Radiography (PA-view and Cuono-view) and MRI were available. During the same period, a control MRI subgroup of the first 37 males (17 right and 20 left wrists) and 20 females (9 right and 11 left wrists), was selected from all the patients without symptoms of carpal boss. 
Patients with previous carpal boss surgery were excluded.

The complete group was initially reviewed by the two readers in random order. Finally, a consensus reading was accomplished.

The Cuono view (modified lateral view with the hand slightly $\left(30-40^{\circ}\right)$ supinated and in a $20-30^{\circ}$ ulnar deviation) is considered as the most reliable projection to demonstrate the bony prominence $(2,3)$ and was used as reference.

All MRI examinations were performed on a 1.5 Tesla MRI scanner (Aera -Siemens /

Erlangen, Germany) using a dedicated wrist coil. The MRI protocol is summarized in Table 1. The images were displayed with a medical monitor, three Megapixel Barco display, 20", 2048 x 1536 resolution (Barco / Kortrijk, Belgium).

All carpal boss patients were symptomatic for at least two months. Age, gender, and side of involvement were analyzed. On radiography and MRI, the presence of an os styloideum, the precise location and exact bony morphology of the carpal boss and the different possible quadrangular joint components were investigated in anatomical detail. The Minnaar coalition classification (4) (type 1: fibrous; type 2: partial osseous bridge; type 3: complete osseous bridge; type 4: type 3 with other carpal anomalies) was used. The presence of bone marrow edema (BME) - noticed as a high signal area around (a part of) the quadrangular joint on FST2-WI - was assessed and correlated with the presence of pain. Any additional osseous and soft tissue abnormalities on MRI were noted.

The same parameters (except for gender and side as they were matched) were analyzed in the control subgroup.

\section{Statistical analysis}

The data was processed using SPSS Statistics -version 23 (IBM, Chicago, USA). Inter- and intra-observer agreements were assessed with Cohen 's kappa ( $\kappa)$ test. Interpretation of the 
coefficients was as follows: poor $(<0)$, slight $(0-0.20)$, fair $(0.21-0.40)$, moderate $(0.41-0.60)$, substantial (0.61-0.80), and almost perfect (>0.80). The two tailed Fisher's exact test was used for comparison of categorical variables (presence or absence of BME and pain), with pvalues $<0.05$ considered as statistically significant. The Pearson product-moment correlation $(0$ - 1) determined the relation between BME and pain. The independent-samples T-test was used to assess the equality of the means and the Levene 's test to evaluate the equality of variances of age between the subgroups.

\section{Results}

Our series included 37 males (65\%) and 20 females (35\%). The mean age was 42,7 years, the median age 42 years, with a distribution ranging from 12 to 77 years (Table 2).

Radiography revealed a carpal boss in 44 patients (77\%) and MRI in an additional 13 (23\%). In $13 / 57$ patients (23\%) an os styloideum (Fig. 1 and 2) was found, of which two small ones were not visible on radiography. Moreover a coalition Minnaar type 1 was found on MRI between the third metacarpal (MC) and an os styloideum in two patients .

The exact bony location and morphology was clearly demonstrated in all cases by MRI, whereas radiography mostly failed to reveal this because of bony superposition. A total of 60 abnormal joint morphologies in 57 patients was observed on MRI: a carpal boss was located at the third carpometacarpal joint (28 patients), between the os styloideum and the third MC (12 patients), at the second carpometacarpal joint (7 patients), at the process of the third MC (6 patients), between the dorsal side of an anatomical variant between the trapezoid bone and the third MC (3 patients), at the second MC-os capitatum joint (1 patient), at the joint between the second and third MC base (1 patient), at the os styloideum-os capitatum-third MC joint (1 patient), or at the trapezoid-capitate bone synostosis (1 patient). Two patients showed a double aberrant joint form and one had an accessory bony coalition. 
Bone marrow edema on MRI was present in 16/57 patients $(28.1 \%)$. The interobserver agreement concerning the presence of BME was substantial $(\kappa=0.73$ for the first and 0.80 for the second reading). The intra-observer values were almost perfect for the first reader $(\kappa=$ $0.92)$ and substantial for the second reader $(\kappa=0.71) .14(87,5 \%)$ of the 16 carpal boss with BME were diagnosed by radiography and an additional 2 (12.5\%) by MRI. However, 39 patients $(68.4 \%)$ showed no BME (30 with carpal boss both on standard radiographs and MRI and 11 only on MRI). Pain was present in $18 / 57$ patients (31.6\%), whereas 38/57 patients (66.7\%) had no pain. The clinical data from 1 patient (1.7\%) was unclear. BME was demonstrated more often in younger patients (Table 2), which is suggestive for a (micro)traumatic rather than a degenerative etiology. The bony abnormality in case of BME was localized in 8 patients at the os styloideum-third carpometacarpal joint $(8 / 13 ; 62 \%)$, in 5 at the third carpometacarpal joint $(5 / 28 ; 18 \%)$, in 2 at the second carpometacarpal joint $(2 / 7$; $29 \%$ ) and in one at the joint between the second and third MC base. The carpal boss in the two patients in which the second carpometacarpal joint was involved, was only visible by revealing BME at the os styloideum on MRI. BME at the os styloideum was believed to be caused by a recent trauma in one patient (tennis player) and by chronic hyperextension in another patient (Fig. 2). BME, correlated with a painful dorsal prominence at presentation (Table 3), showed a true positive correlation in 11/15 (73\%) and a true negative correlation in 34/41 (83\%) patients, which is highly significant (Fischer 's exact test: $\mathrm{p}<0.001)$. There was a positive correlation between BME and pain (Pearson 's $\mathrm{r}(56)=0.632$, which is significant at the 0.01 level (two-tailed)).

Old traumatic lesions at the MC base were observed in 4 patients and only one patient presented with a recent fracture.

Degeneration was present in 27 cases (47 \%) with the typical dorsal "volcano"- type osteophytes (Fig. 3A). A large intra-osseous ganglion was found in one case. 
Only 4/57 (7\%) patients presented with a ganglion. Interference of the carpal boss with the normal path of the m. extensor carpi radialis brevis (Fig. 3B) resulted in tendinosis in 4/57 (7\%) patients. One patient had a dorsal ligament rupture (Fig. 3C). Finally one clinically diagnosed dorsal soft tissue mass because of active synovitis, was illustrated by contrast enhancement (Fig. 3D).

In the series without carpal boss symptoms, only one patient had a Minnaar type 2 coalition (trapezoid-capitate bone), without surrounding BME. No bony or soft tissue anomalies were discovered. The difference of mean age between the control subgroup $(\mathrm{M}=28.3, \mathrm{SD}=1.3)$ and the carpal boss subgroup $(\mathrm{M}=42.7, \mathrm{SD}=16.9)$ was significant $(\mathrm{t}(84.410)=-5.485, \mathrm{p}<$ $0.001)$ and Levene 's test indicated unequal variances $(F=14.260, p<0.001)$.

\section{Discussion}

As initially described, a carpal boss appears almost exclusively in the area of the carpometacarpal joint (5) - called the quadrangular joint - where the metacarpal bases, the dorsal carpometacarpal joint ligaments, and the insertion of the extensor carpi radialis tendons are closely related (Fig. 3E). The bony contours and ligaments (Fig. 4) (6) restrict the range of motion (less than $\left.5^{\circ}\right)(7)$. Our analysis confirmed that carpal boss was most prevalent at the third (49\%) and second carpometacarpal joint (12\%), which is in line with the literature. However, MRI also illustrated in $39 \%$ of the patients a carpal boss in a number of variable locations.

According to the literature, a ganglion is present in up to $30 \%$ (8), although in our series only in $7 \%$.

The os styloideum - described by Saltzmann (2) - is an accessory bone (9) between the trapezoid and capitate bones and the second and third MC (8). It originates from a separate ossification center (10). The prevalence is estimated between $1-4 \%(3,11,12)$. It can be 
isolated (2\%), but mostly fused, typically to a metacarpal (94\%) (2). Since the dorsal ligaments are absent in the presence of an os styloideum (13), it is hypothesized that carpal boss represents a congenital coalition $(5,7)$, which can cause symptoms in case of a fracture of a fibrous coalition (4). Carpal coalitions were only reported in the area of the second and third carpometacarpal joint in a cadaveric study $(5,7)$. There is also a contrasting high prevalence of an os styloideum in carpal boss (33\% in the literature and $23 \%$ in our series) compared to normal wrists (1-3\% in the literature, $0 \%$ in our control group) (11). Some authors therefore prefer the term carpal boss syndrome for the association of an os styloideum and wrist pain (14).

In our series, BME was localized more often at the os styloideum-third carpometacarpal joint and almost two thirds of the patients with an os styloideum showed BME. Carpal boss can be caused by either a stress-induced bony hypertrophy or by healing micro-fractures, typically resulting from repetitive forced extension $(15,16)$. The major axes of stress on the wrist run through the scapholunate and the quadrangular joint (17). Only one patient had a bony coalition containing BME at the third carpometacarpal joint $(18,19)$.

The traumatic etiology (23-27\%) (15) includes metacarpal base fractures, dorsal ligamentous avulsions, avulsion fractures at the extensor tendon insertion, and direct lesions of the os styloideum. The bony prominence can also be caused by a non-united fracture. In athletes, hand injuries are frequent ( 3 to $9 \%$ of all injuries). They often occur in golf and racquet sports (3) and in collision sports, the incidence can rise up to $15 \%$ (20). A case report describes an acute carpal boss in a swimmer due to a tear of a dorsal ligament (21). Acute BME after a direct injury of the os styloideum was described in an ice hockey player (15) and ( similar to one of our cases) in a tennis player (22). Old traumatic lesions were only shown in 4/57 patients (7\%). Chronic symptoms can be related to joint degeneration, an inflamed ganglion, a bursa or an extensor tendinopathy. Osteophytes related to a secondary localized 
degenerative osteoarthritis can cause a carpal boss. They were a frequent finding (47\%) in our series.

Carpal boss usually presents in the fourth decade. The mean age of our group was 42,7 years, the median age 42 years with a broad age distribution. However, some authors noticed earlier cases (23), even in adolescents (13), as we also noticed in our series. The literature does not describe a gender predominance, although we noticed a male to female ratio of almost 2/1. According to literature carpal boss is present in up to $8-26 \%$ of the general population and it is a bilateral finding in up to $21 \%$ (24). Symptomatic carpal boss is mostly found in the dominant hand $(2,25)$, although that finding was not confirmed in our series. Most protuberances are ganglions with only cosmetic complaints. An insidious pain can be aggravated by activity (26) or be related to degenerative osteoarthritis, bursitis or tendon subluxation (27). Especially repetitive forced extension aggravates the symptoms because of an insertion tenosynovitis. Carpal boss of either etiology limits the lateral movements or causes subluxation of the extensor tendons (10).

The presence of an os styloideum alone does not allow for confirmation of the diagnosis of symptomatic carpal boss as the incidence in the carpal boss population is $8-26 \%$, with less than $3 \%$ being symptomatic (23).

The diagnosis of carpal boss and the presence of an os styloideum can be made on plain radiographs, although bony superposition from the surrounding carpal bones can impede the evaluation of the exact morphology and the detection of an os styloideum, as illustrated in our series in $2 / 13$ ossa styloidei ( $15 \%)$.

A ganglion can appear as a soft tissue mass without calcifications (23) on radiography. MRI is of additional value to detect ganglions in up to $30 \%$ (23).

Especially 3D-GRE isotropic sequences can clearly demonstrate an accessory ossicle or a morphological anomaly, although without the use of radiation, compared to CT. 
A diffuse signal increase in case of symptomatic BME of an os styloideum or around the quadrangular joint is well depicted on fluid sensitive sequences (SE T2-WI FS, STIR). The absence of BME seems to have a high negative predictive value $(83 \%)$ and the presence has a less positive predictive value $(73 \%)$ (Fischer's exact test: $p=0.0002)$.

There can be a joint effusion in the carpometacarpal joint or thinning of the cartilage. Dorsal osteophytes (even small ones) and subchondral cysts are clearly visualized.

Dorsal soft tissue lesions - difficult to illustrate with CT - and edema can be illustrated with MRI. Tears of the dorsal ligaments can be revealed, although additional MR midcarpal arthrography may be required to illustrate these subtle lesions. The disruption of the attachment of the extensor tendon(s) and an avulsion fracture at their insertion can be revealed, although small chip fragments may be missed and (cone beam)CT could be necessary.

A fracture at the base of the metacarpals is usually clearly seen on MRI, also because of the absence of bony superposition (similar to CT) and the accompanying BME (not visible on CT). The exact location and morphology of the carpal boss can allow for a more limited resection or shaving in order to prevent ligamentous post-operative instability. Future research needs to determine in which cases these additional bony and soft tissue MRI abnormalities can alter the therapy or clinical outcome.

We acknowledge our study has some limitations as the study has a retrospective design. There is also a statistical significant difference in age between the subgroups. However as BME seems to be more frequent in younger patients with pain and the control subgroup is composed of an even younger population without signs of BME, this can be neglected. The illustration of those various findings in this - as far as we know first - global MRI analysis of the quadrangular joint can call for further attention, especially in patients with persistent symptoms. 
In conclusion, the presence of bony anomalies on radiography does not allow for correlation with the patient 's symptoms, neither does the absence of bony anomalies allows excluding the diagnosis of carpal boss. CT allows for detection of subtler bony changes, but the correlation with symptoms remains elusive. MRI can illustrate a variable bony morphology in detail and also a variety of additional bony and soft tissue diseases causing carpal boss.

BME around the quadrangular joint does highly correlate with a painful carpal boss.

\section{Acknowledgements}

The authors thank Mehdi Ghafariyan and Sander Oogink for their statistical assistance.

\section{Conflict of interest}

The authors declare that there are no conflicts of interest. 


\section{References:}

1. Fiolle J. Le carpe bossu. Bull Mem Soc Nat Chir 1931;57:1687-1690.

2. Conway WF, Destouet JM, Gilula LA et al. The carpal boss: an overview of radiographic evaluation. Radiology 1985; 156:29-31.

3. Park MJ, Namdari S, Weiss AP. The carpal boss: review of diagnosis and treatment. J Hand Surg 2008;33A:446-449.

4. Mespreuve M, Vanhoenacker F, Verstraete K. Lunotriquetral coalition, a normal variant that may rarely cause ulnar sided wrist pain. JBSR 2015; 98:72-78.

5. Alemohammad AM, Nakamura K, El-Shenway M et al. Incidence of carpal boss and osseous coalition: an anatomic study. J Hand Surg 2009;34:1-6.

6. Nakamura K, Patterson RM, Viegas SF. The ligament and skeletal anatomy of the second through the fifth carpometacarpal joints and adjacent structures. J Hand Surg 2001; 26:10161028.

7. Gunther SF. The carpometacarpal joints. Orthoped Clin North Am 1984; 15:259-277.

8. Hultgren T, Lugnegard H. Carpal boss. Acta Orthop Scandinavica 1986; 57:547-550.

9. Kalantari BN, Seeger L, Motamedi K et al. Accessory ossicles and sesamoid bones: spectrum of pathology and imaging evaluation. Applied Radiology 2007; 10: 28-37.

10. Kootstra G., Huffstadt AJ, Kauer JM. The styloid bone, a clinical and embryological study. The Hand 1974; 6: 185-188.

11. Cuono CB, Watson HK. Carpal boss: surgical treatment and etiological considerations. Plast Reconstr Surg 1979; 63:88-93.

12. Köhler A, Zimmer EA. Grenzen des Normalen und Anfänge des Pathologischen im Röntgenbild des Skeletts. Stutgart: George Thieme Verlag, 1989: 80-103.

13. Capo JT, Orillaza N.S, Lim PK. Carpal boss in an adolescent: case report. J Hand Surg 2009; 34:1808-1810. 
14. Apple JS, Martinez S, Nunley JA. Painful os styloideum: bone scintigraphy in carpe bossu disease. Am J Roentgenol 1984; 142:181-182.

15. Kissel P. Conservative management of symptomatic carpal bossing in an elite hockey player: a case report. J Can Chiropr Assoc 2009; 53:282-289.

16. Vermeulen GM, de With MC, Bleys RL et al. Carpal boss: effect of wedge excision depth on third carpometacarpal joint stability. J Hand Surg 2009; 34:7-13.

17. Tang JB. General concepts of wrist biomechanics and a view from other species. J of Hand Surg (Eur) 2008;33:519-525.

18. Geutjens G. Carpal bossing with capitate-trapezoid fusion- a case report. Acta Orthop Scand 1994.65:97-98.

19. Tielliu IF, Wellen PA. Carpal boss caused by an accessory capitate. Case report. Acta Orthop Belg 1998; 64:107-108.

20. Rettig AC. Athletic injuries of the wrist and hand. Am J Sports Med 2003; 31:1038-1049. 21. Maquirriain J, Ghiss JP. Acute os styloideum injury in an elite athlete. Skeletal Radiol 2006; 35:394-396.

22. Gomes AF, Paganella VC, Paganella MC. Computed tomography and magnetic resonance imaging findings of os styloideum in a symptomatic athlete. Radiol Bras 2010; 43:207-209.

23. Hunt TR. Degenerative and post-traumatic arthritis affecting the carpometacarpal joints of the fingers. Hand Clin 2006; 22:221-228.

24. Nguyen V, Choi J, Davis KW. Imaging of wrist masses. Curr Probl Diagn Radiol 2004; 33:147-160.

25. Fusi S, Watson HK, Cuono CB. The carpal boss: a 20-year review of operative management. J Hand Surgery 1995; 20:405-408.

26. Lawson JF. Symptomatic radiographic variants in extremities. Radiology 1985:157:625631. 
27. Stein JM, Cook TS, Simonson S et al. Normal and variant anatomy of the wrist and hand on MR imaging. Magn Reson Imaging Clin N Am 2011; 19:595-608.

Table 1: MR imaging protocol.

\begin{tabular}{|c|c|c|c|c|c|c|}
\hline \multicolumn{7}{|c|}{ MR imaging protocol } \\
\hline Pulse sequence & $\begin{array}{l}\text { FOV } \\
(\mathrm{cm})\end{array}$ & $\begin{array}{l}\text { Slice } \\
\text { thickness } \\
(\mathrm{mm})\end{array}$ & Matrix & $\begin{array}{l}\text { TR } \\
(\mathrm{msec})\end{array}$ & $\begin{array}{l}\mathrm{TE} \\
(\mathrm{msec})\end{array}$ & Nex \\
\hline Coronal SE T1-WI & 12 & 2 & $512 \times 384$ & 479 & 22 & 1 \\
\hline Coronal SE PD-T2 FS & 12 & 2 & $256 \times 180$ & 3500 & $26 / 91$ & 3 \\
\hline Axial TSE PD FS & 10 & 3 & $320 \times 256$ & 1950 & 27 & 2 \\
\hline 3D T2*- GRE (DESS) & 12 & 0,5 & $256 \times 230$ & 24,31 & 8,31 & 1 \\
\hline
\end{tabular}

(Contrast series can be added in case of erosions or synovitis)

Abbreviations: (T)SE: (turbo)spin echo, PD: proton density, 3D-GRE: three dimensional gradient echo recalled echo, FS: fat saturation, FOV: field of view, TR: repetition time, TE: echo time, Nex: number of excitations.

Table 2: Age distribution of the patients (years) and of the patients with BME on MRI.

\begin{tabular}{|ccccccccc|}
\hline & $<20 \mathrm{y}$ & $20-29 \mathrm{y}$ & $30-39 \mathrm{y}$ & $40-49 \mathrm{y}$ & $50-59 \mathrm{y}$ & $\geq 60 \mathrm{y}$ & $12-77 \mathrm{y}$ \\
\hline Number & 3 & 10 & 13 & 13 & 9 & 9 & 57 \\
\hline $\begin{array}{c}\text { Number } \\
\text { with BME }\end{array}$ & 0 & 7 & 4 & 3 & 1 & 1 & 16 \\
\hline
\end{tabular}


Table 3: Bone marrow edema (BME) in patients with pain at the second or third CAPOMETACARPAL JOINT: patients with pain (SY) and without pain (AS) at the quadrangular joint.

\begin{tabular}{|lccclccc|}
\hline $\begin{array}{l}\text { PAT } \\
(\mathrm{n})\end{array}$ & BME & BME & TOTAL $(\%)$ & BME & BME & TOTAL \\
\hline SY & 11 & 7 & 18 & SY & $61 \%$ & $39 \%$ & $32 \%$ \\
\hline AS & 4 & 34 & 38 & AS & $11 \%$ & $89 \%$ & $66 \%$ \\
\hline unclear & 1 & 0 & 1 & unclear & $100 \%$ & $0 \%$ & $2 \%$ \\
\hline TOTAL & 16 & 41 & 57 & TOTAL & $28 \%$ & $72 \%$ & $100 \%$ \\
\hline
\end{tabular}

Fischer's exact test: $p=0.0002$ 


\section{FIGURES:}

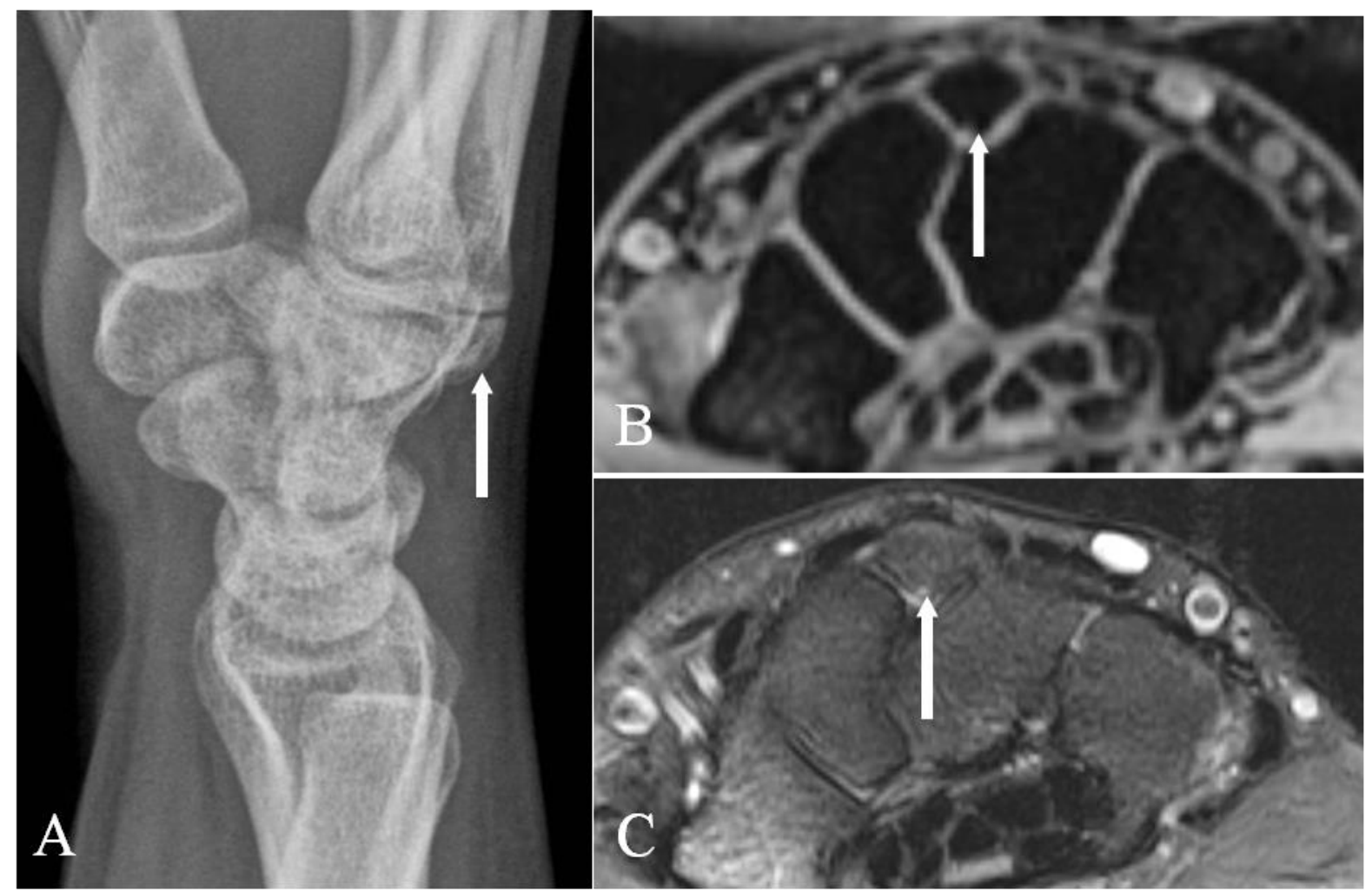

Fig.1. - Os styloideum. (A) Plain radiography Cuono- view, (B) 3D-GRE and (C) Coronal SE T1-WI.

(A) The modified profile Cuono view can illustrate the presence of an os styloideum (arrow).

(B-C) MRI clearly shows the os styloideum (arrows). The exact morphology, localization and the signal intensity $(\mathrm{C})$ of the bone, the bone marrow and the adjacent structures can be illustrated as well. 


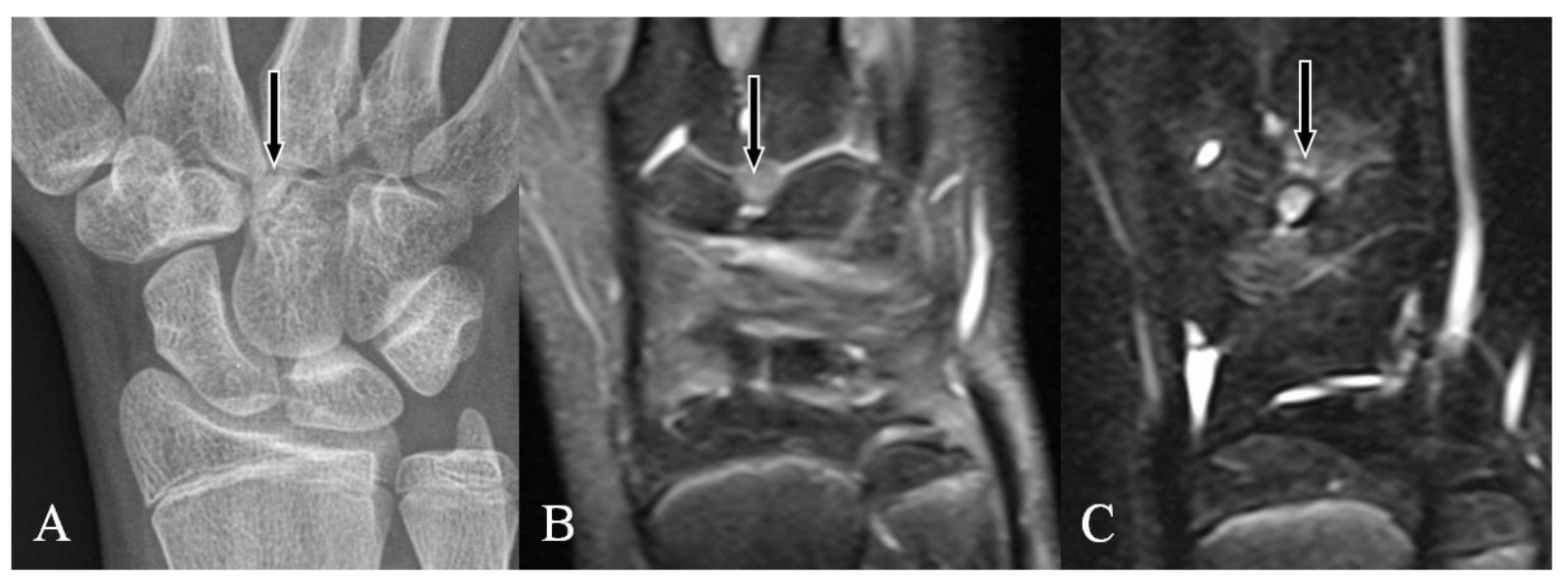

Fig. 2. - CB presenting with pain after repetitive stress at the $\mathrm{CMC}$-joint in a professional piano player. (A) Plain radiography PA view, (B) Coronal SE PD-WI FS and (C) SE T2-WI FS.

(A) Presence of an os styloideum (arrow).

(B-C) Focal bone marrow edema at the os styloideum (arrows) and the base of MC-III (C) (arrow). The pain disappeared after resection of the os styloideum. 


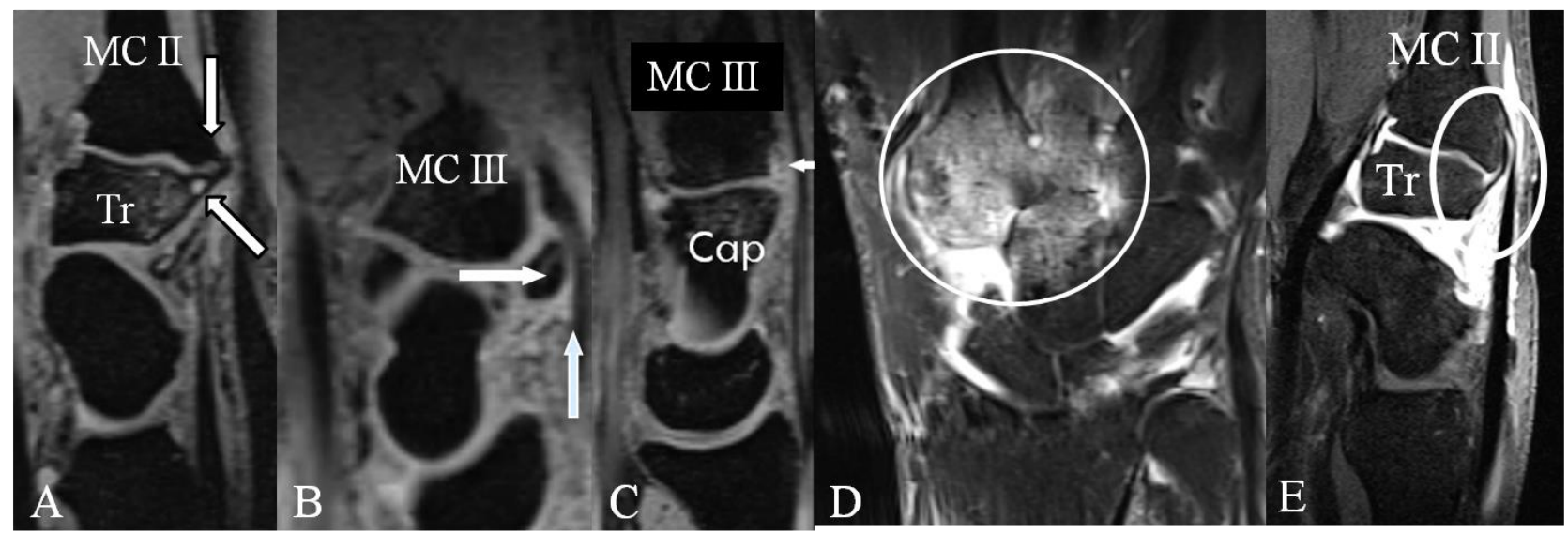

Fig. 3. - (A, B and C) Sagittal 3D-GRE (D) Coronal SE T1-WI FS with gadolinium contrast, and (E) MR midcarpal arthrography sagittal SE T1-WI FS.

(A) Osteophytes (arrows) resembling a volcano, with subchondral cyst (oblique arrow) and irregular narrowing of the dorsal joint space.

(B) $\mathrm{CB}$ due to an os styloideum (horizontal arrow) interfering with the normal path of the ECRB tendon (vertical arrow).

(C) Rupture of the dorsal CMC-III ligament (arrow) with surrounding soft tissue edema.

(D) Contrast enhancement in CB due to rheumatoid arthritis. Dorsal prominent synovitis (arrows) at the CMC-III joint.

(E) Trapezoid-MC-II joint showing the close relationship (oval) of the dorsal CMC-ligament and the more superficial tendon of the ECRL. 


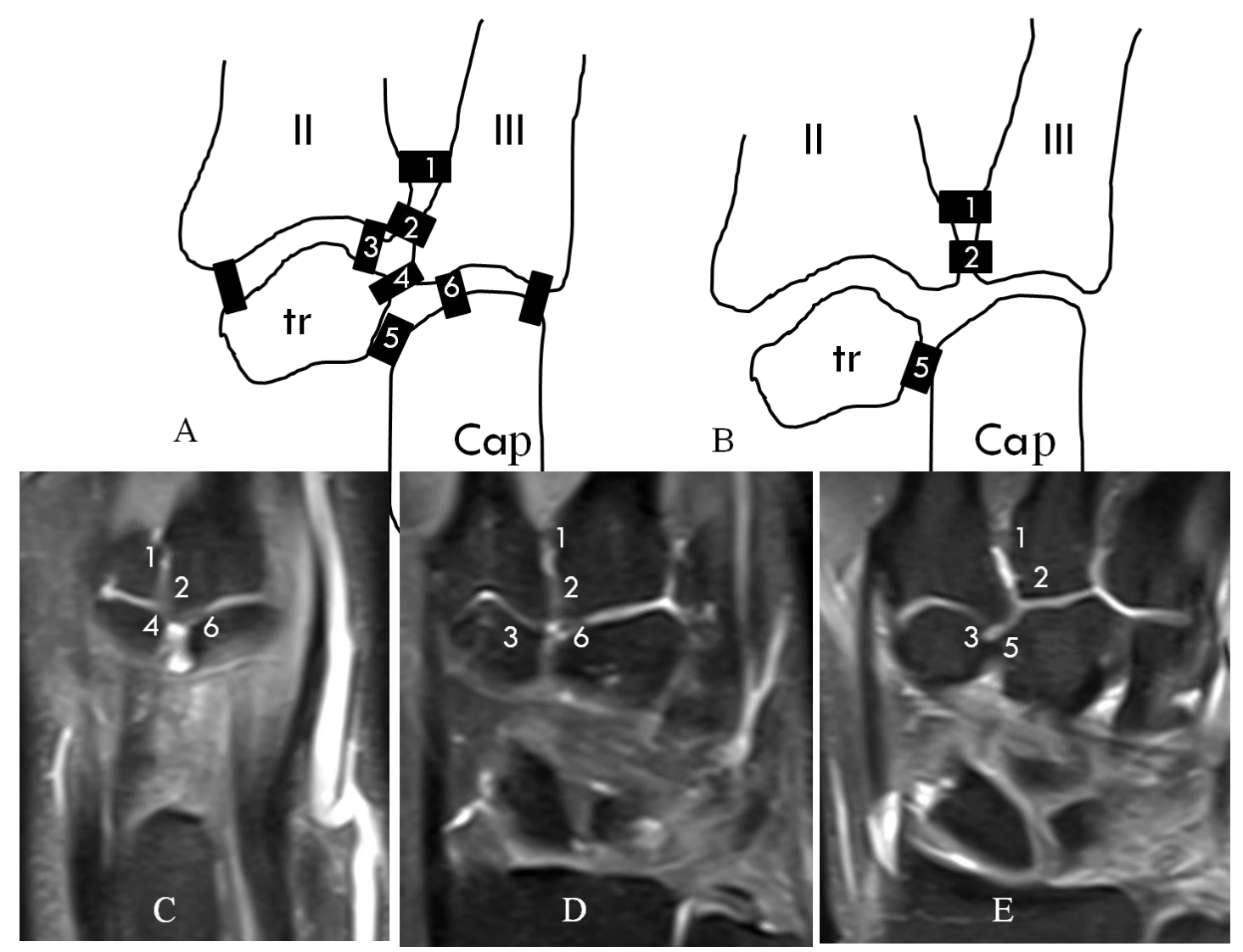

Fig. 4. - Normal ligamentous anatomy of the dorsal side around the quadrangular joint. (AB) schematic drawing, (C-E) Coronal SE PD-WI FS from dorsal to more palmar.

(A) most dorsal position with prominent base of MC-III, (B) more palmar slice level with prominent base of MC-II, (C-E) ligaments 1. distal between the base of II and III, 2. proximal between the base of II and III, 3. between tr and II, 4. between tr and III, 5 . between tr and Ca and 6. between III and $\mathrm{Ca}$

(II = metacarpal 2, III = metacarpal 3, Ca = capitate bone, $\operatorname{tr}=$ trapezoid bone $).$ 